\title{
Characterising resuscitation promoting factor fluorescent-fusions in mycobacteria
}

\author{
Iria Uhía, Nitya Krishnan and Brian D. Robertson*
}

\begin{abstract}
Background: Resuscitation promoting factor proteins (Rpfs) are peptidoglycan glycosidases capable of resuscitating dormant mycobacteria, and have been found to play a role in the pathogenesis of tuberculosis. However, the specific roles and localisation of each of the 5 Rpfs in Mycobacterium tuberculosis remain mostly unknown. In this work our aim was to construct fluorescent fusions of $M$. tuberculosis Rpf proteins as tools to investigate their function.

Results: We found that Rpf-fusions to the fluorescent protein mCherry are functional and able to promote cell growth under different conditions. However, fusions to Enhanced Green Fluorescent Protein (EGFP) were non-functional in the assays used and none were secreted into the extracellular medium, which suggests Rpfs may be secreted via the Sec pathway. No specific cellular localization was observed for either set of fusions using time-lapse video microscopy.

Conclusions: We present the validation and testing of five M. tuberculosis Rpfs fused to mCherry, which are functional in resuscitation assays, but do not show any specific cellular localisation under the conditions tested. Our results suggest that Rpfs are likely to be secreted via the Sec pathway. We propose that such mCherry fusions will be useful tools for the further study of Rpf localisation, individual expression, and function.
\end{abstract}

Keywords: Rpfs, Mycobacteria, Tuberculosis, Fluorescent fusions, Microscopy

\section{Background}

Resuscitation promoting factor proteins (Rpfs) are peptidoglycan glycosidases able to resuscitate dormant bacteria. After the first description in Micrococcus luteus [1], five homologs were found in Mycobacterium tuberculosis, which can reactivate growth of dormant mycobacteria [2,3]. They share a conserved Rpf domain, a 70 amino-acid region responsible for biological activity [4]. Knockouts of these proteins have been extensively studied [5-7]. The muralytic activity of these proteins $[8,9]$ may serve in peptidoglycan remodelling, although it is also proposed that the muropeptides produced modulate innate immune responses in the host, or activate cell resuscitation pathways [2, 10-12]. Despite the importance of these proteins in resuscitation of the important pathogen M. tuberculosis, little is known about their individual, specific roles, or localisation in the cell. Structural studies on $\mathrm{RpfB}, \mathrm{E}$ and $\mathrm{C}$ catalytic domains shows a high degree of similarity, but also differences among

\footnotetext{
* Correspondence: b.robertson@imperial.ac.uk

MRC Centre for Molecular Bacteriology and Infection, Department of Medicine, Imperial College London, London SW7 2AZ, UK
}

these five proteins [13-16] . All Rpfs are expressed in early exponential and resuscitation phases, but are differentially expressed under stress [17], possibly due to differential regulation [18-22], suggesting their roles do not completely overlap.

Putative signal sequences at the amino terminus suggests they function extracellularly [23]. RpfB and RpfE interact with the cell wall hydrolase RipA, and RpfB and RipA colocalise at the septum of dividing cells, suggesting RpfB-RipA interactions are involved in separation of daughter cells during reactivation [12, 24-26].. The localisation and specific roles of the other Rpfs is not clear. Purified RpfE induces maturation of dendritic cells in mice, and it was suggested (but not demonstrated) that other Rpfs have this activity [27]. RpfA, RpfC and RpfE were found in culture filtrates of M. tuberculosis [28, 29], showing they are, at least in part, secreted into the extracellular medium, where they might exert autocrine and/or paracrine signalling functions. RpfC has also been found in membranes $[29,30]$ suggesting multiple locations. Recently, His-tagged M. tuberculosis RpfA, RpfB, RpfD and RpfE overproduced in M. smegmatis were detected in the culture supernatant by ELISA [31]. 
Fusion of Rpfs to fluorescent proteins would help localise them within the cell and give indications about function and possible distinct roles. To date only the localisation of RpfB fused to RFP has been communicated [24]. In the work reported here, we tested fusions of the five M. tuberculosis Rpfs to two different fluorescent proteins, EGFP and mCherry. We found that all fusions to mCherry, but none of the EGFP fusions, were functional. These results make Rpf-mCherry fusions interesting tools for studying resuscitation in mycobacteria.

\section{Results}

\section{Fusion of Rpfs to fluorescent proteins and microscopic} analysis

\section{EGFP fusions}

We amplified the five rpf genes from $M$. tuberculosis $\mathrm{H} 37 \mathrm{Rv}$ and constructed C-terminal translational fusions to EGFP. This way, the $\mathrm{N}$-terminus of each $r p f$ gene was unmodified, maintaining putative signal sequences. We transformed $M$. smegmatis $\mathrm{mc}^{2} 155$, a fast growing nonpathogenic mycobacteria used as a surrogate for $M$. tuberculosis, with plasmids harbouring each of the five constructs. The strains producing Rpf-EGFP fusions grew at the same rate as those expressing EGFP alone (Additional file 1: Figure S1a). Each Rpf-EGFP protein was expressed from a replicative plasmid (pSTetRO$r p f A / B / C / D / E$-egfp $)$ under control of a tetracycline inducible promoter.

Using static microscopy the five fusion proteins appeared distributed along the cell body, but in a fraction of cells RpfB-EGFP, RpfC-EGFP and RpfE-EGFP localised at the tips and septum, the only areas of peptidoglycan production and growth in mycobacteria (Fig. 1). However, only around $12 \%$ of cells showed this localisation $(12.8 \%$ for RpfB-EGFP $(n=219), 12.7 \%$ for RpfC-EGFP $(n=134)$ and $11.5 \%$ for RpfE-EGFP $(n=139))$, raising the question whether this was due to over-production of the fusion proteins, although RpfB has been reported to localise to the septum [24].

We were unable to see any evidence of localisation of the proteins using time-lapse microscopy of cells growing in a microfluidic chamber; instead fluorescence was distributed along the cell body (Additional file 2: Movie S1). We could see transient accumulations of fluorescence at the septum in some cells, but in other cells, it became apparent that the accumulation of fluorescence at the tips or septum was most likely caused by precipitated proteins since these cells did not grow (Additional file 3: Movie S2).

All Rpfs have predicted signal peptides at the $\mathrm{N}$ terminus [23], and are most probably secreted by the general secretion $(\mathrm{Sec})$ pathway. Consequently, proteins must be completely folded in the periplasm or cell wall area [32]. However, EGFP is likely to be inefficiently

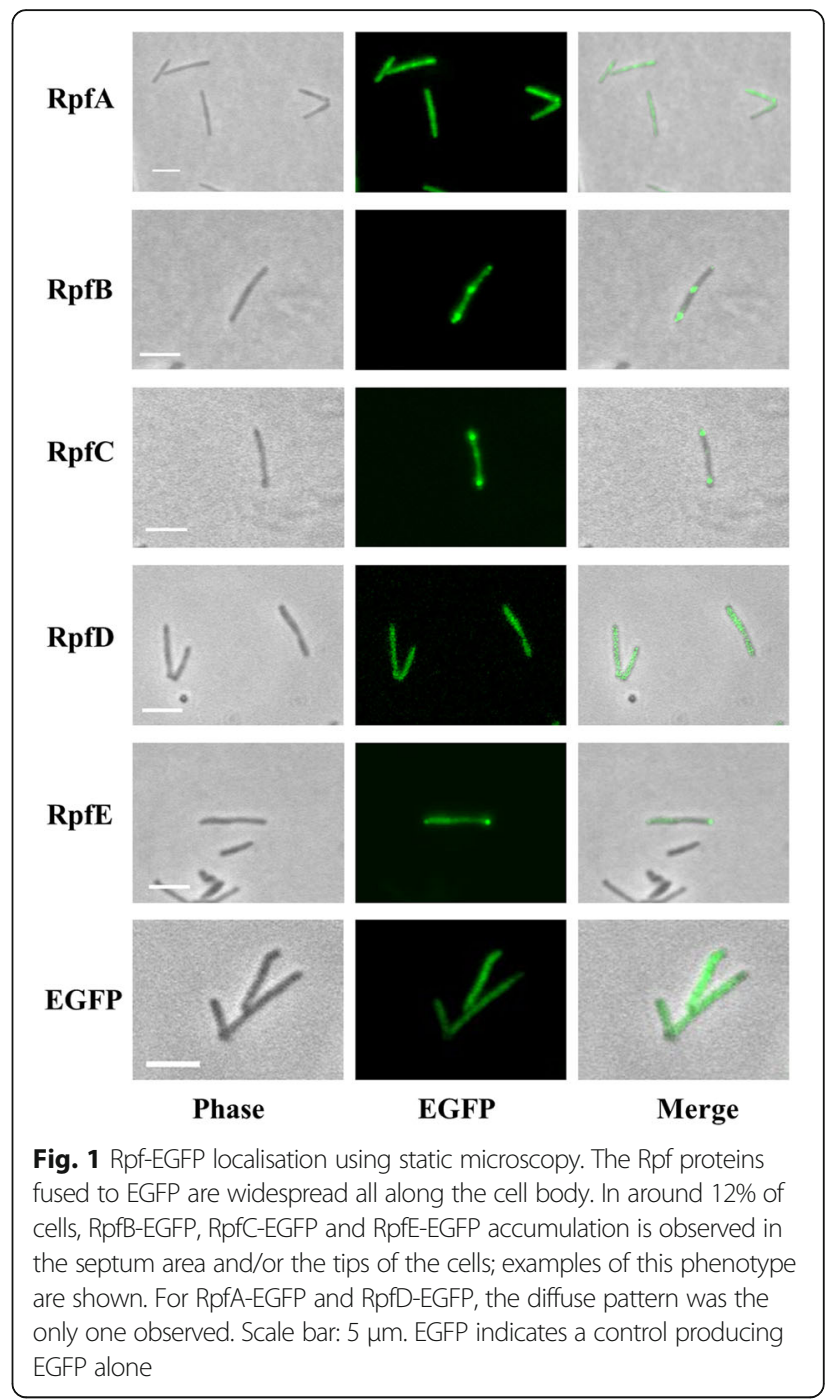

folded in this location, due to the presence of cysteine groups able to form interchain disulphide bonds in this oxidizing environment, therefore blocking its maturation [33]. This in turn probably affects the localisation and activity of EGFP-fused Rpfs. Therefore, as an alternative, we fused the Rpfs to mCherry, a fluorescent protein that does not contain cysteine residues and hence should not be misfolded in the periplasm.

\section{mCherry fusions}

The rpf genes were fused to the $\mathrm{N}$-terminus of mcherry and the fluorescent fusion proteins produced from replicative plasmids pMEND - $r p f A / B / C / D / E$-mcherry, under control of a tetracycline inducible promoter.

M. smegmatis transformed with each of the plasmids grows at the same rate as the control strain (Additional file 1: Figure S1b) and static microscopy did not show a specific localisation for any of the Rpfs; all of them diffused throughout the cell body (Fig. 2). In time lapse 


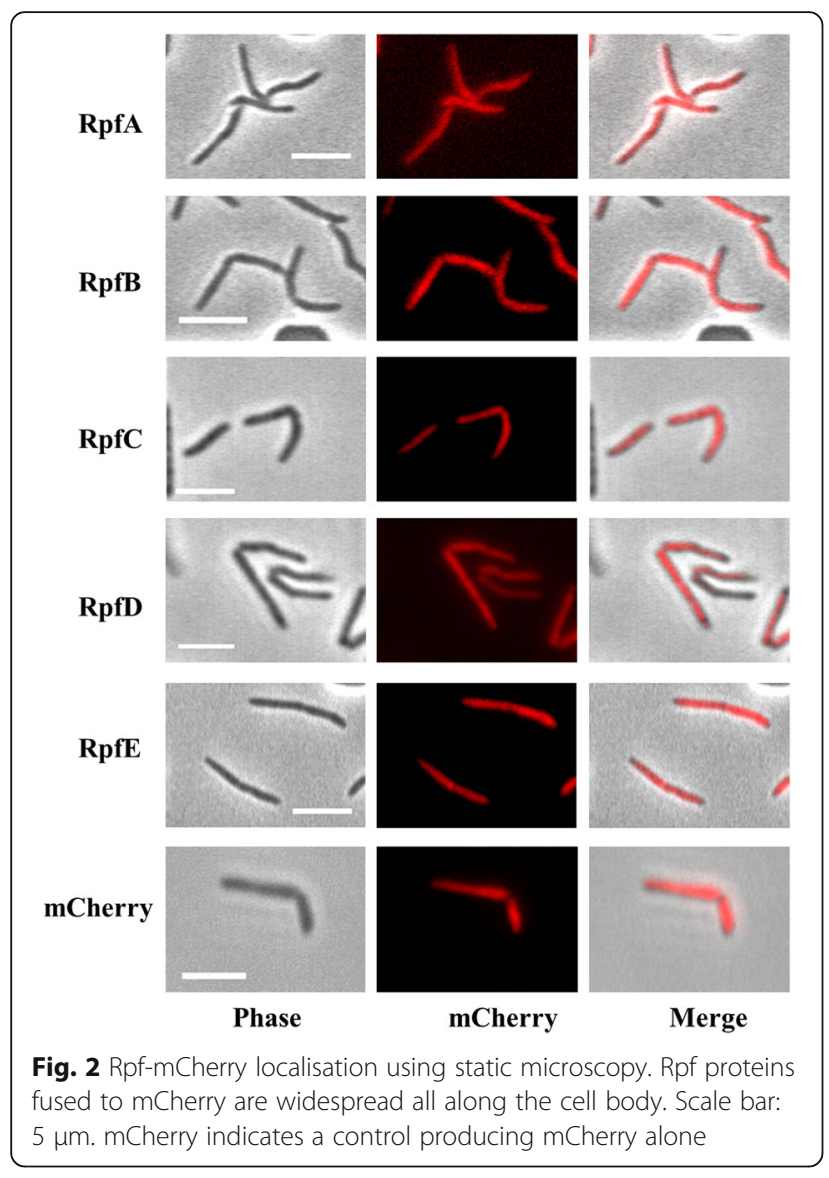

microscopy the results were very similar. As with EGFP fused proteins, we could sometimes see temporary accumulations of fluorescence in the septum area prior to division, especially for strains producing RpfA, RpfB and RpfE (Additional file 4: Movie S3).

\section{Western blots of fluorescent fusion proteins}

Western blot was used to check the presence of fusion proteins in soluble extracts and precipitated fractions of disrupted cells, and in culture supernatants. Using an anti-mCherry antibody, we were able to detect proteins bands in soluble extracts and the precipitated fraction that matched the expected size for each fusion (Fig. 3a). Some of the proteins appear as multiple bands, probably due to protein degradation/aggregation. When we analysed the filtered culture supernatant, we were able to detect RpfAmCherry and RpfE-mCherry proteins (Fig. 4a), confirming that these are secreted into the extracellular medium, as had previously been shown by proteomics and ELISA [28, 29, 31].

The detection of RpfA-mCherry and RpfE-mCherry in culture supernatants strongly suggests that these mCherry-fusions are efficiently translocated to their correct localisation in the cell. In contrast, when analysing Rpf-EGFP fusions, none were detected in the culture supernatant (Fig. 4b) and neither RpfC-EGFP nor RpfD-EGFP were detected in soluble and precipitated extracts (Fig. 3b), supporting our hypothesis that these fusions have impaired the function of the Rpf protein.

\section{Functionality of Rpf-mCherry fusions}

We tested the functionality of the fusions by checking the ability of each strain to resume growth after being subjected to nutritional shift-down, adapted from a published protocol [4]. Briefly, strains were grown in rich broth until stationary phase; then 100-600 cells per $\mathrm{ml}$ were subcultured in defined minimal broth. The lag phase of the cultures was substantially reduced when any of the five Rpfs-mCherry fusions was produced, in comparison to the strain producing mCherry alone (Fig. 5a), achieving OD values close to stationary phase (3) before the control starts to grow. There is some biological variability regarding the time when the mCherry strain begins to grow, ranging from 40 to $100 \mathrm{~h}$ after the Rpf-mCherry strains, but the results are highly reproducible. However, the mCherry strain does eventually reach the same OD, despite the longer lag phase and there is no statistical difference. Interestingly, the enhancement of growth was equal for all the overproduced Rpfs. A reduction in the lag phase was not observed for the EGFP-fused Rpfs (Fig. 5b). The growth curves shown are in this case a representative of at least 3 biological replicates for each strain and not mean values, as the different strains start to grow in a highly stochastic fashion in each experiment, with the control strain (EGFP) the first to grow. These results strongly suggest that Rpfs-EGFP fusions are not functional: growth inhibition due to overproduction of Rpfs-EGFP is unlikely; both sets of proteins (Rpfs-mCherry and Rpfs-EGFP) are under the control of the same promoter and induced under the same conditions, and western blot analysis shows the Rpfs-EGFP proteins are not produced in higher amounts.

\section{Functionality of Rpf-mCherry fusions under different types of stress}

In addition to demonstrating that Rpf-mCherry fusions are functional in a nutritional shift-down assay, we also tested if their overproduction could resuscitate cells after they had been subjected to different stresses. We aimed to determine if specific Rpfs were required for particular stresses, or whether there are functional overlaps that might form a hierarchy.

Suboptimal growing conditions or the presence of antibiotics produce non-platable mycobacteria, which resume growth in a Rpf-dependent manner [21, 31, 34]. The different strains were subjected to acidic ( $\mathrm{pH} 4.5$ ) or osmotic stress conditions $(1 \mathrm{M} \mathrm{NaCl})$, or nutritional 

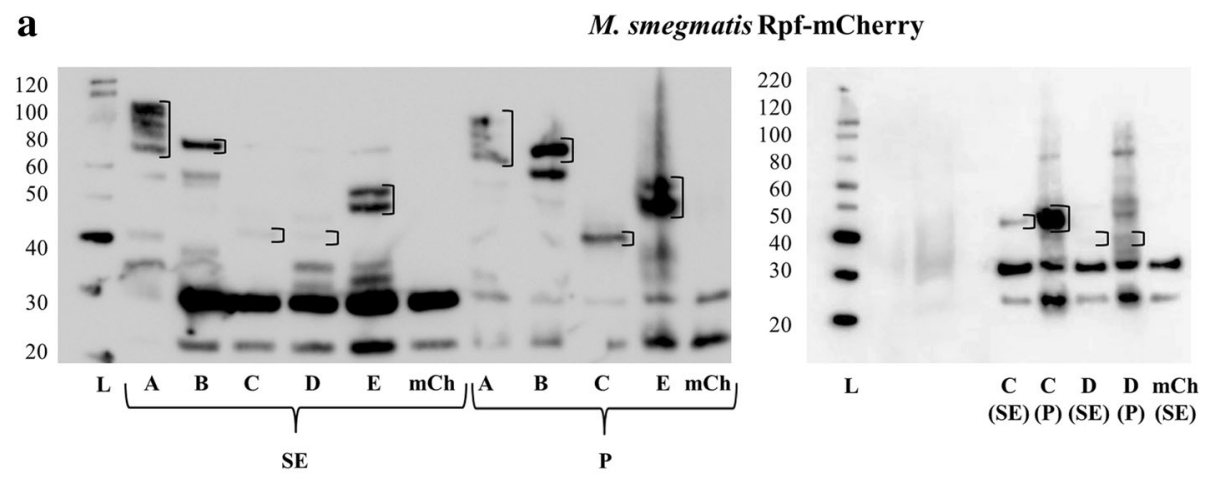

b

M. smegmatis Rpf-EGFP
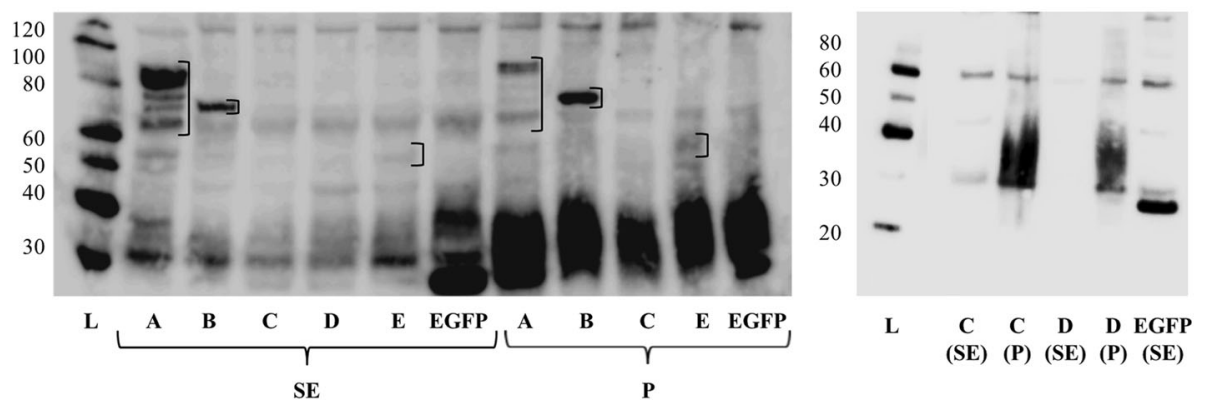

Fig. 3 Localisation of Rpf-mCherry/EGFP fusions by western blot: protein extracts. The samples were extracted as detailed in Methods. SDS-PAGE gels were loaded with approximately $20 \mu \mathrm{g}$ of soluble extract (SE; total proteins) and $5 \mu \mathrm{l}$ of precipitated extract $(\mathrm{P})$, previously resuspended in $50 \mu \mathrm{l}$ of loading buffer. The letters A-E correspond to samples from RpfA-E-mCherry/EGFP-producing cultures, respectively. mCh and EGFP refer to the control strains harbouring control plasmids. The molecular weight ladder $(L)$ values in $\mathrm{kDa}$ are shown on the left of each gel. Empty squares have been included encircling the bands of interest. a The left western blot performed with Rpfs-mCherry producing strains presents bands at the expected heights for all the fusion proteins in the SE and P. In some cases (RpfA and RpfE) more than one band appears per sample, most probably due to protein degradation/aggregation. The right blot contains repeat RfpC-mCherry samples and RpfD-mCherry pellet (that is not present on the left). The results show that these two proteins are present in both SE and P, but are more concentrated in the P fraction. $\mathbf{b}$ In the left blot performed with Rpfs-EGFP producing strains, RpfC-EGFP could not be identified in the SE or P. The right blot contains repeat RfpC-mCherry samples (as this protein could not be identified on the left) and RpfD-mCherry pellet (that was not present in the WB on the left). These two proteins could not be identified in the SE or P. Hypothetical molecular weight of the fusion proteins: RpfA-EFFP/mCherry $(\approx 67 \mathrm{kDa})$, RpfB-EGFP/mCherry $(\approx 65 \mathrm{kDa})$, RpfCEGFP/mCherry ( $\approx 45 \mathrm{kDa})$, RpfD-EGFP/mCherry $(\approx 43 \mathrm{kDa})$, RpfE-EGFP/mCherry $(\approx 46 \mathrm{kDa})$

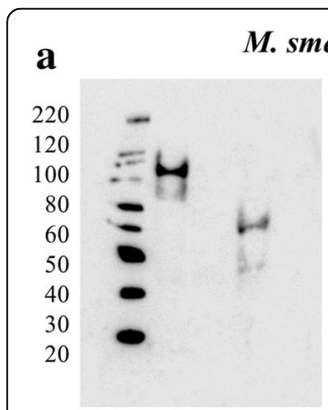

$\mathbf{L} \quad \mathbf{A} \quad \mathbf{B} \quad \mathbf{E}$

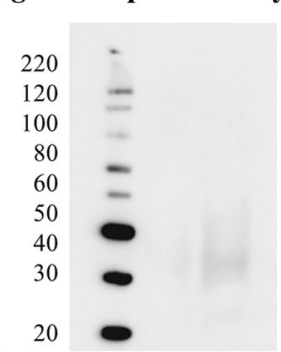

L C C D

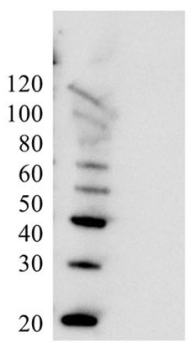

$\mathrm{L} \mathbf{m C h}$

\section{b}

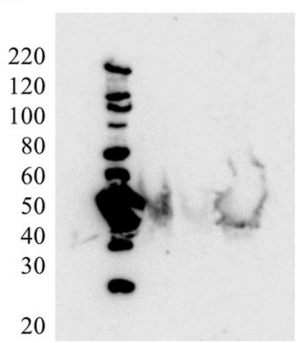

$\mathbf{L} \quad \mathbf{A} \quad \mathbf{B} \quad \mathbf{E}$

\section{M. smegmatis Rpf-EGFP}

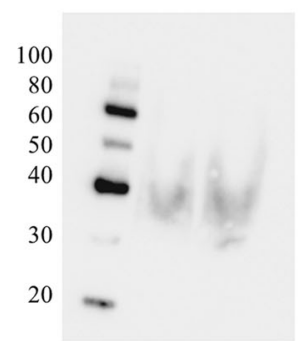

L $\quad$ C $\quad$ D

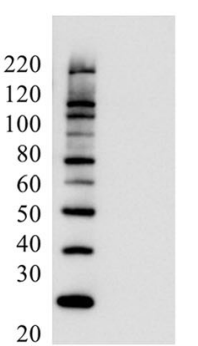

L EGFP

Fig. 4 Localisation of Rpf-mCherry/EGFP fusions by western blot: culture supernatants. SDS-PAGE gels were loaded with $20 \mu$ l of concentrated culture supernatants (previously precipitated and resuspended in $40 \mu \mathrm{l}$ of loading buffer; see Methods). The letters A-E correspond to samples from RpfA-EmCherry/EGFP-producing cultures, respectively. $\mathrm{mCh}$ and EGFP refer to the control strains expressing fluorophore alone. The molecular weight ladder $(\mathrm{L})$ values in $\mathrm{kDa}$ are shown on the left of each gel. a The western blots performed with culture supernatants of RpfA-mCherry and RpfE-mCherry producing strains present bands at the expected heights for the fusion proteins, as seen in soluble and precipitated extracts (Fig. 3). b In the western blots performed with culture supernatants of Rpf-EGFP producing strains, none of the samples present bands at the expected heights, indicating that probably none of the fusion proteins is being secreted to the extracellular medium. Hypothetical molecular weight of the fusion proteins: see Fig. 3 legend 


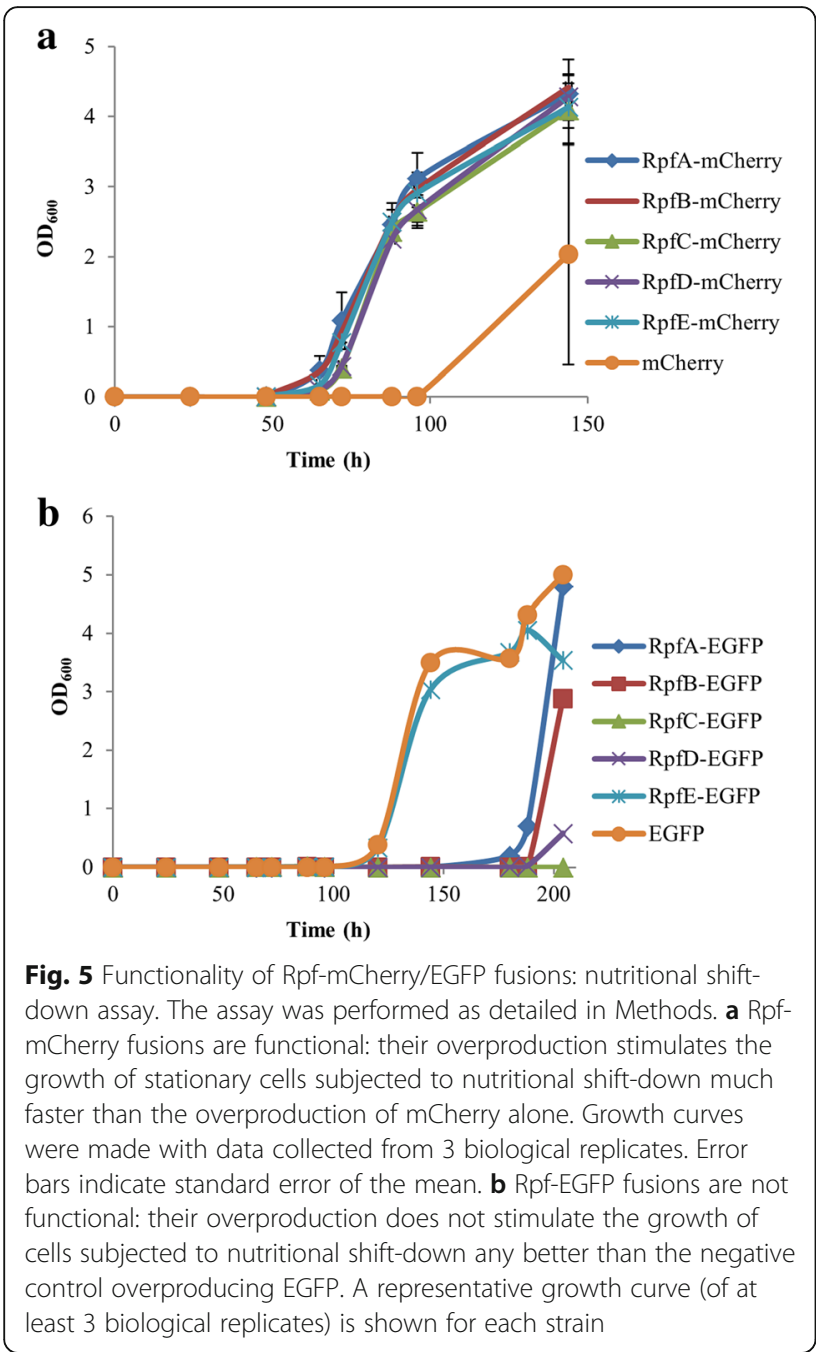

starvation (incubation in PBS), after which the capacity to stimulate the growth of non platable cells was calculated for each strain using the Most Probable Number (MPN) method (US Food and Drug Administration, 2010), and Colony Forming Units (CFU) counts.

As expected, cells under acidic, osmotic stress and nutrient starvation exhibit a less significant loss of viability (tested by CFUs) than those exposed to antibiotic stress [31], with a CFU loss of 2-6 fold for Phosphate Buffered Saline (PBS) and $1 \mathrm{M} \mathrm{NaCl}$, and 3-13 fold for $\mathrm{pH} 4.5$ after 6 days of incubation (data not shown). 6 days was chosen as the analysis point, since the number of platable cells remains stable for up to 7 additional days after this point, indicating that the cells are no longer dying due to the stress.

MPN and CFU values were analysed after stress. Cells previously exposed to acidic conditions ( $\mathrm{pH} 4.5$ ) show a tendency to increased capacity to resume growth of non platable cells when overproducing any of the RpfmCherry fusions compared to mCherry alone (Fig. 6a),

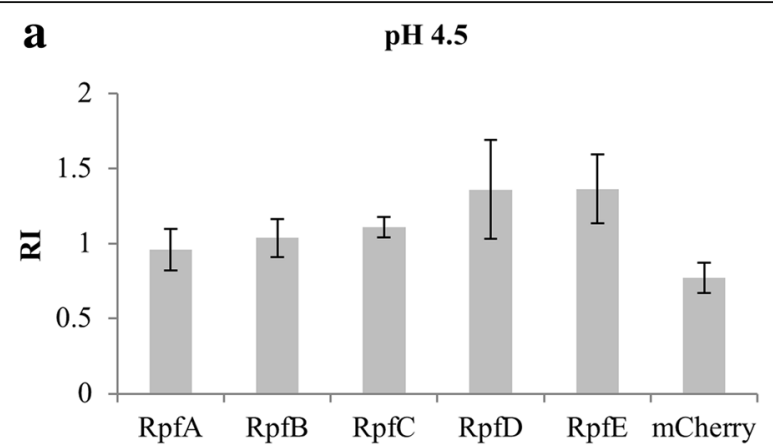

b

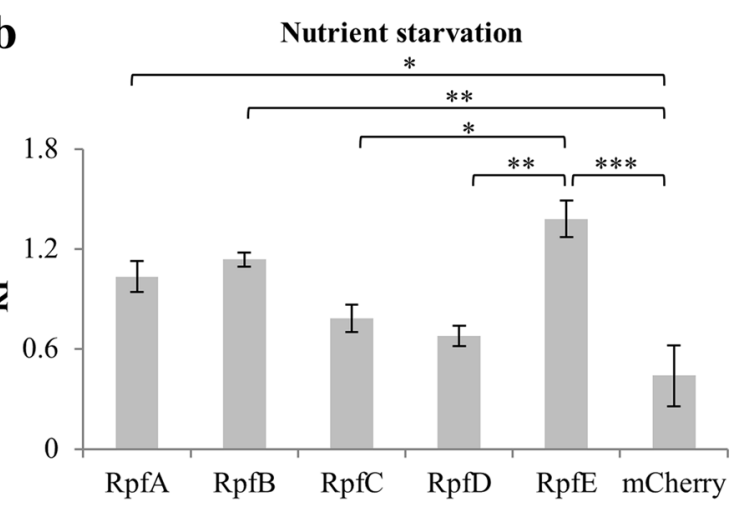

c

$1 \mathrm{M} \mathrm{NaCl}$

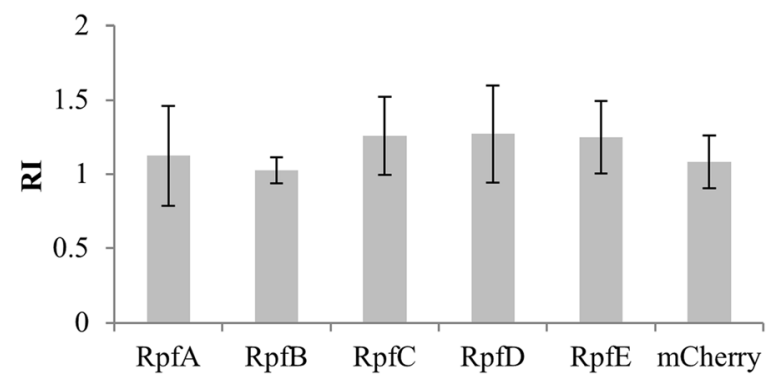

Fig. 6 Induction of the growth of non platable cells by Rpf-mCherry producing strains after different stress conditions. The strains were subjected to three stress conditions and subsequently the potential for resuscitation of non platable cells was calculated for each strain as detailed in Methods and expressed as the Resuscitation Index (RI): $\log _{10}(\mathrm{MPN})-\log _{10}(\mathrm{CFU})$. a Strains overproducing RpfD-mCherry and RpfE-mCherry show enhanced growth of non platable cells produced under pH 4.5, but this is not statistically significant. b The strain overproducing RpfE-mCherry enhances the growth of non platable cells produced under nutrient starvation to a greater extent, followed by RpfB-mCherry and RpfA-mCherry. c None of the strains overproducing Rpf-mCherry enhances the growth of non platable cells produced under $1 \mathrm{M} \mathrm{NaCl}$. Three independent experiments were performed for each condition and strain. Error bars indicate standard error of the mean. Statistical differences in RI between strains were calculated using One Way Analysis of Variance followed by Bonferroni t-test. Cases in which differences were found to be statistically significant, are indicated by asterisks: ${ }^{* *} P<0.001$; ${ }^{*} P<0.01 ;{ }^{*} P<0.05$ 
with the highest numbers for the strains overproducing RpfE-mCherry and RpfD-mCherry (however the differences where not statistically significant by One Way Analysis of Variance under the conditions tested). This is in agreement with previous work that demonstrated that RpfE and RpfD are the two Rpfs with highest mRNA levels under acidic conditions [17]. In the case of nutrient starvation, RpfE-mCherry was again best at promoting growth of non platable cells, followed by RpfB and RpfA, the results being in this case statistically significant (Fig. 6b). For osmotic stress, there was no difference between the control strain and the overproducing strains (Fig. 6c).

\section{Discussion}

The cellular localisation of four of the five Rpfs in $M$. tuberculosis remains unknown. As a prelude to investigating this, we have constructed and tested the functionality of two sets of Rpf fluorescent fusion proteins. Rpf-EGFP fusions gave a diffuse pattern of localisation when analysed using microscopy, with some of the proteins (RpfB-EGFP, RpfC-EGFP and RpfE-EGFP) showing a tendency to aggregate. None of the five EGFP- fusion proteins were functional, with none of them secreted into the extracellular medium; this suggests that Rpfs are secreted by the Sec pathway and therefore folded in the periplasmic area, where EGFP is known to be misfolded [33].

Rpf-mCherry fusions also did not show specific localisation in the cell, but in contrast to EGFP-fusions, none formed aggregates. Western blot showed all Rpf-mCherry proteins were found in the soluble and insoluble extracts of disrupted cells, with RpfAmCherry and RpfE-mCherry also found in the culture supernatant. However, we were unable to detect any other Rpf proteins in the supernatant, contrary to published studies $[28,29,31]$. The explanation for this may be the different methodologies used. The fact that overproduced RpfA and RpfE were previously found to be the most abundant Rpfs in culture supernatants by Enzyme Linked Immunosorbent Assay (ELISA) [31], together with our results suggests that they may be the main Rpfs functioning extracellularly, whereas the rest may, at least transiently, attach to the cell envelope to exert their function. Producing Rpf-mCherry from their chromosomal loci with native promoters would give a more exact picture of the real situation in the cell. Tracking fluorescence production during infection or exposure to different stresses would also help better understand the role of these proteins, and expression from the native Rpf loci or from an integrative plasmid might help to obtain detailed localisation for each of these proteins. mCherry has potential for single-molecule localisation microscopy (SMLM) [35], but photoactivatable mCherry (PAmCherry) may be more suitable for photoactivated localisation microscopy (PALM) [36].

Rpf-mCherry proteins were all functional in terms of equal capacity to promote growth-recovery in a nutritional shift-down assay. Since all the Rpf-fusions were functional, we tested their capacity to promote growth of the non platable cells created under stress conditions. RpfE-mCherry and RpfD-mCherry seem to be most effective after $\mathrm{pH} 4.5$ stress, and RpfE-mCherry after starvation stress; it has been suggested that this type of activity could be associated with tuberculosis reactivation [37]. The loss of platable cells (CFU) is low under these stress conditions, making interpretation difficult and differences between groups are not always statistically significant. We also used a parental strain producing native levels of Rpfs, and cannot rule out that they may interfere with testing of the contribution of individual overexpressed Rpf-mCherry fusions. The ideal would be to repeat these experiments with a parental strain deprived of the $5 \mathrm{Rpfs}$, but this is not currently available to us.

\section{Conclusions}

In conclusion, the Rpf-mCherry fusions described here are a useful tool for the study of mycobacterial resuscitation factors, and their role in the biology of these organisms.

\section{Methods}

DNA manipulation, bacterial strains and growth conditions

The strains and the plasmids used in this work are listed in Additional file 5: Table S1. Primers used in this work are listed in Additional file 6: Table S2. Mycobacterium smegmatis $\mathrm{mc}^{2} 155$ and derivatives were grown in defined liquid medium, either Middlebrook 7H9 (OADC supplemented, Difco), Sauton's medium $(0.05 \%$ potassium phosphate monobasic, $0.05 \%$ magnesium sulphate, $0.2 \%$ citric acid, 0 . $005 \%$ ferric ammonium citrate, $0.4 \%$ L-asparagine, 5\% glycerol, $1 \%$ zinc sulphate solution; $\mathrm{pH}$ adjusted to 7 ) or Hartmans-de Bont minimal medium (Hartmans et al, 2006), all supplemented with $0.025 \%$ tyloxapol (Sigma). Liquid cultures were grown aerobically at $37{ }^{\circ} \mathrm{C}$ in an orbital shaker at $180 \mathrm{rpm}$. For growth on solid medium mycobacteria were grown on Middlebrook 7H11 agar (OADC supplemented, Difco) incubated at $37^{\circ} \mathrm{C}$. When needed, hygromycin $(50 \mu \mathrm{g} / \mathrm{ml})$ and kanamycin $(20 \mu \mathrm{g} / \mathrm{ml})$ were used for plasmid selection and maintenance. Tetracycline ( $5 \mathrm{ng} / \mathrm{ml}$ ) was used to induce mCherry (and fusion derivatives) production. Escherichia coli DH5 $\alpha$ strain was used as a host for cloning. It was grown in LB medium at $37{ }^{\circ} \mathrm{C}$ in an orbital shaker at $180 \mathrm{rpm}$. LB agar plates were used. Hygromycin $(150 \mu \mathrm{g} / \mathrm{ml})$ and kanamycin $(50 \mu \mathrm{g} / \mathrm{ml})$ were used for plasmid selection and maintenance. 


\section{Construction of plasmids}

Plasmids harbouring each $r p f$ gene fused to EGFP were constructed by amplifying each of the five $r p f$ genes in M. tuberculosis $\mathrm{H} 37 \mathrm{Rv}$ genome (rpfA (Rv0867c), rpfB (Rv1009), rpfC (Rv1884c), rpfD (Rv2389c) and rpfE (Rv2450c)) and cloning them in the EcoRI site of plasmid pST5552 [38]. In-frame fusions to EGFP were checked by sequencing. The promoter region of pST5552 was substituted by cloning the tetracyclineinducible promoter from plasmid pMEND [39] in the BamHI-EcoRI sites to generate plasmids pSTetRO-rpfA/ $B / C / D / E$-egfp.

For the generation of plasmids with rpfs fused to mCherry protein, the five amplified genes were cloned into the BamHI-NdeI sites of pMEND-mCherry [40], which produced the fusion to the C-terminus of mCherry. A RBS was included in the $5^{\prime}$ primer upstream of each $r p f$ start codon. The resulting plasmids pMEND$r p f A / B / C / D / E$-mcherry have the fusion genes under the control of a tetracycline promoter.

Plasmids were electroporated into competent $M$. smegmatis $\mathrm{mc}^{2} 155$ as described [41].

\section{Microscopy}

Microscopy was performed in the Facility for Imaging by Light Microscopy (FILM) at Imperial College London. Time-lapse live cell microscopy was performed in B04A plates with an ONIX flow-control system (Merck-Millipore). Cells were loaded in the chamber at an $\mathrm{OD}_{600}$ of 0.1 from mid-exponential cultures in Hartmans-de Bont medium, and cultured at a continuous flow rate $(1 \mathrm{psi})$ in a temperaturecontrolled chamber at $37{ }^{\circ} \mathrm{C}$. Fluorescent fusion proteins were induced with tetracycline before loading the cells in the microfluidic chamber, where they continued to be perfused with the inducer. Images were captured every 15 min using a Zeiss Axiovert 200 inverted widefield microscope fitted with an EMCCD (C9100-02) camera (Hammamatsu) controlled by HCImage software, using a $63 \mathrm{X}$ objective. Z-stacks were collected at $1 \mu \mathrm{m}$ intervals to ensure in-focus images were collected. Images were analysed using Fiji image processing software [42].

For static microscopy, $20 \mu \mathrm{l}$ samples of growing cultures were mounted on slides using Mowiol 4-88 (Calbiochem) previously to their visualization.

\section{Western blotting}

For western blots of soluble extracts and precipitated fraction of disrupted cells, $10 \mathrm{ml}$ cultures of the strains were grown in Hartmans-de Bont broth with the inducer tetracycline up to an $\mathrm{OD}_{600}$ of 1 . The cultures were then centrifuged and resuspended in $500 \mu \mathrm{lBS}+$ protease inhibitor (cOmplete, Sigma) and disrupted in a water bath sonicator. The soluble extract and the precipitated fraction were subsequently separated by centrifugation and protein concentration in the soluble extract was determined using the Pierce ${ }^{\mathrm{Tw}}$ BCA Protein Assay Kit (Thermo Fisher). An equal amount of soluble proteins for all the samples was loaded in the gels for western blotting. The precipitated fractions were resuspended in the same volume for all the samples and a fixed volume was loaded on the gels. Western blots were performed using the NuPAGE Western blotting system (Thermo Fisher). SeeBlue Pre-stained Protein Standard (Thermo Fisher) was used as size standard. As a primary antibody, polyclonal rabbit anti-EGFP antibody (Thermo Fisher, dilution 1:1000) or polyclonal rabbit anti-mCherry antibody (Novus Biologicals, dilution 1:1000) were used, and as a secondary antibody we used goat anti-rabbit IgGHRP (Santa Cruz Biotechnology, dilution 1:5000). The blots were developed using the SuperSignal West Femto Substrate Trial Kit (Thermo Fisher) in a LAS-3000 Fuji Imager.

For the western blots of filtrated culture supernatants, $100 \mathrm{ml}$ of each strain were grown in Hartmans-de Bont broth without tween 80 and with the inducer tetracycline up to an $\mathrm{OD}_{600}$ of approximately 0.6. Protease inhibitor was added to the cultures $30 \mathrm{~min}$ before collecting, centrifuging and filtering the supernatants $(0$. $22 \mu \mathrm{m})$. The proteins in the supernatants were concentrated by Ion-Exchange chromatography, adapting a published protocol [4]. The pI of the Rpfs-mCherry and Rpfs-EGFP fusion proteins was theoretically calculated using the Compute $\mathrm{pI} / \mathrm{Mw}$ tool on the ExPASy Server [43]. It was found in all cases to be below the $\mathrm{pH}$ of the buffers (7.5) so the anion exchanger DEAE-Sepharose was used. $2 \mathrm{ml}$ columns were washed with $5 \times$ volumes of water and then equilibrated with $5 \times$ volumes of buffer A $(20 \mathrm{mM}$ TrisHCl, pH 7.5; $20 \mathrm{mM} \mathrm{KCl,} 1 \mathrm{mM}$ EDTA, $1 \mathrm{mM} \mathrm{DTT})$. The supernatants were passed through the column and eluted with $3 \times$ volumes of buffer B (buffer A with $1 \mathrm{M} \mathrm{NaCl}$ ). $2 \mathrm{ml}$ samples of the first eluate were precipitated with $20 \%$ TCA and washed twice with acetone, dried and resuspended in loading buffer for its use in western blot, which was performed as previously explained.

The theoretical molecular weight of the fusion proteins was calculated using the Compute $\mathrm{pI} / \mathrm{Mw}$ tool on the ExPASy Server [43].

\section{Nutritional shift-down assay}

Strains were grown in $7 \mathrm{H} 9$ broth for 4 days in shaking, ensuring they reached stationary phase. They were then subcultured in $10 \mathrm{ml}$ Hartmans-de Bont broth in the presence of the inducer tetracycline. The number of bacteria inoculated in Hartmans-de Bont was checked by CFUs counting and was determined to be between 
100 and 600 cells/ $\mathrm{ml}$ in all cases. Cultures were grown in shaking and $\mathrm{OD}_{600}$ measured over time. Three biological replicates of each strain were analysed.

\section{Exposure to stress and calculation of viable counts}

$M$. smegmatis strains harbouring each Rpf-mCherry or $\mathrm{m}$-Cherry alone were grown in shaking up to mid-log phase $\left(\mathrm{OD}_{600} \approx 0.6\right)$ in Sauton's medium before being exposed to different stress conditions. For osmotic stress, a $\mathrm{NaCl}$ solution in water was added to the cultures at a $1 \mathrm{M}$ final concentration. For acidic stress, cultures were washed in pH 4.5 Sauton and resuspended in the same medium. For nutrient starvation, cultures were washed twice in PBS-0.025\% Tyloxapol (Sigma) and resuspended in the same solution. Samples for CFU counts were taken at this point and subsequently, the cultures were incubated for 6 days at $37{ }^{\circ} \mathrm{C}$ in static. After the incubation, the cultures were washed and resuspended in Sauton's medium, and samples were taken for CFU and MPN counts and resuscitation index calculations.

Assessment of CFU counts was performed in serial dilutions of cultures in triplicate by using the standard droplet method [44] and subsequent incubation of the agar plates by $48 \mathrm{~h}$.

For MPN counts, serial dilutions of $200 \mu \mathrm{l}$ cultures samples were incubated in 96-well plates in Sauton's medium containing the inducer tetracycline. Three wells were used for each dilution. The plates where incubated in static at $37^{\circ} \mathrm{C}$ for 2 weeks and MPN calculated [45]. Three independent experiments were performed for each condition and strain. The potential for resuscitation of non platable cells of the different strains was expressed as the resuscitation index (RI): $\log _{10}(\mathrm{MPN})$ $\log _{10}(\mathrm{CFU})$ [31]. Statistical differences in RI between strains were calculated using One Way Analysis of Variance (one-way ANOVA). We subsequently applied Bonferroni t-test, an all pairwise multiple comparisons procedure, to isolate the groups that differ from others. One-way ANOVA and Bonferroni t-test were applied using SigmaPlot (Systat Software, San Jose, CA).

\section{Additional files}

Additional file 1: Figure S1. Growth of M. smegmatis Rpf-EGFP and Rpf-mCherry producing strains in 7H9. (a) Growth curve of strains overproducing Rpf-EGFP proteins and a control strain overproducing EGFP. (b) Growth curve of strains overproducing Rpf-mCherry proteins and a control strain overproducing mCherry. Growth curves were made with data collected from 3 biological replicates. Error bars indicate standard deviation. (PDF $16 \mathrm{~kb}$ )

Additional file 2: Movie S1. Localisation of RpfE-EGFP fusions in M. smegmatis (time lapse microscopy). RpfE-EGFP is shown as an example; all the Rpfs fused to EGFP present widespread localisation. (AVI $168 \mathrm{~kb}$ )

Additional file 3: Movie S2. Putative RpfB-EGFP inclusion bodies in $M$. smegmatis (time lapse microscopy). (AVI $150 \mathrm{~kb}$ )
Additional file 4: Movie S3. Localisation of RpfE-mCherry fusions in M. smegmatis (time lapse microscopy). RpfE-EGFP is shown as an example; all the Rpfs fused to mCherry present widespread localisation. (AVI $351 \mathrm{~kb}$ )

Additional file 5: Table S1. Bacterial strains and plasmids used in this study. (PDF $27 \mathrm{~kb}$ )

Additional file 6: Table S2. Primers used in this study. (PDF 14 kb)

\section{Abbreviations}

CFU: Colony Forming Units; EGFP: Enhanced Green Fluorescent Protein; ELISA: Enzyme Linked Immunosorbent Assay; MPN: Most Probable Number; PALM: Photoactivated localisation microscopy; PBS: Phosphate Buffered Saline; Rpfs: Resuscitation promoting factor proteins; SMLM: Single-molecule localisation microscopy

\section{Acknowledgements}

We would like to acknowledge Debora Keller and Stephen Rothery for providing microscopy support at the Facility for Imaging by Light Microscopy (FILM) (Imperial College London).

\section{Funding}

I.U. was funded by a postdoctoral fellowship from the Fundación Alfonso Martín Escudero and a grant from the Imperial College Trust. The ONIX microfluidics platform was provided by kick-starter funds from the Faculty of Natural Sciences, Imperial College London. The Funders had no role in any aspect of this study.

\section{Availability of data and materials}

All data generated or analysed during this study are included in this published article and its supplementary information files. The datasets used and/or analysed during the current study are available from the corresponding author on reasonable request.

\section{Authors' contributions}

$I U$ and BDR conceived and designed the experiments. IU and NK performed the experiments. IU analysed the data. IU and BDR wrote the paper. All authors read and approved the final manuscript.

\section{Ethics approval and consent to participate}

Not applicable.

\section{Consent for publication}

Not applicable.

\section{Competing interests}

The authors declare that they have no competing interests.

Received: 12 July 2017 Accepted: 15 March 2018

Published online: 12 April 2018

\section{References}

1. Mukamolova GV, Turapov OA, Kazarian K, Telkov M, Kaprelyants AS, Kell DB, et al. The rpf gene of Micrococcus luteus encodes an essential secreted growth factor. Mol Microbiol. 2002;46:611-21. https://doi.org/10.1046/j.13652958.2002.03183.x.

2. Kana BD, Mizrahi V. Resuscitation-promoting factors as lytic enzymes for bacterial growth and signaling. FEMS Immunol Med Microbiol. 2010;58:3950. http://femsim.oxfordjournals.org/content/58/1/39.abstract

3. Uhía I, Williams KJ, Shahrezaei V, Robertson BD. Mycobacterial growth. Cold Spring Harb Perspect Med. 2015;5:a021097. https://doi.org/10.1101/ cshperspect.a021097.

4. Mukamolova GV, Turapov OA, Young DI, Kaprelyants AS, Kell DB, Young M. A family of autocrine growth factors in Mycobacterium tuberculosis. Mol Microbiol. 2002;46:623-35. https://doi.org/10.1046/j.1365-2958.2002.03184.x.

5. Downing KJ, Betts JC, Young DI, McAdam RA, Kelly F, Young M, et al. Global expression profiling of strains harbouring null mutations reveals that the five rpf-like genes of Mycobacterium tuberculosis show functional redundancy. Tuberculosis. 2004;84:167-79.

6. Tufariello JM, Jacobs William RJ, Chan J. Individual Mycobacterium tuberculosis resuscitation-promoting factor homologues are dispensable for 
growth in vitro and in vivo. Infect Immun. 2004;72:515-26. https://doi.org/ 10.1128/IAl.72.1.515-526.2004

7. Kana BD, Gordhan BG, Downing KI, Sung N, Vostroktunova G, Machowski $\mathrm{EE}$, et al. The resuscitation-promoting factors of Mycobacterium tuberculosis are required for virulence and resuscitation from dormancy but are collectively dispensable for growth in vitro. Mol Microbiol. 2008;67:672-84. https://doi.org/10.1111/j.1365-2958.2007.06078.x.

8. Mukamolova GV, Murzin AG, Salina EG, Demina GR, Kell DB, Kaprelyants AS, et al. Muralytic activity of Micrococcus luteus Rpf and its relationship to physiological activity in promoting bacterial growth and resuscitation. Mol Microbiol. 2006;59:84-98. https://doi.org/10.1111/j.1365-2958.2005.04930.x.

9. Telkov MV, Demina GR, Voloshin SA, Salina EG, Dudik TV, Stekhanova TN, et al. Proteins of the Rpf (resuscitation promoting factor) family are peptidoglycan hydrolases. Biochemist. 2006;71:414-22. https://doi.org/10. 1134/S0006297906040092.

10. Jo E-K. Mycobacterial interaction with innate receptors: TLRs, C-type lectins, and NLRs. Curr Opin Infect Dis. 2008;21:279-86. http://journals.Iww.com/coinfectiousdiseases/Fulltext/2008/06000/Mycobacterial_interaction_with_ innate_receptors_.11.aspx

11. Nikitushkin VD, Demina GR, Shleeva MO, Kaprelyants AS. Peptidoglycan fragments stimulate resuscitation of "non-culturable" mycobacteria. Antonie Van Leeuwenhoek. 2013;103:37-46. https://doi.org/10.1007/s10482-0129784-1.

12. Nikitushkin VD, Demina GR, Shleeva MO, Guryanova SV, Ruggiero A, Berisio $R$, et al. A product of RpfB and RipA joint enzymatic action promotes the resuscitation of dormant mycobacteria. FEBS J. 2015;282:2500-11. https:// doi.org/10.1111/febs.13292.

13. Squeglia F, Romano M, Ruggiero A, Vitagliano L, De Simone A, Berisio R. Carbohydrate recognition by RpfB from Mycobacterium tuberculosis unveiled by crystallographic and molecular dynamics analyses. Biophys J. 2013;104: 2530-9.

14. Mavrici D, Prigozhin DM, Alber T. Mycobacterium tuberculosis RpfE crystal structure reveals a positively charged catalytic cleft. Protein Sci. 2014;23:4817. https://doi.org/10.1002/pro.2431.

15. Chauviac F-X, Robertson G, Quay DHX, Bagneris C, Dumas C, Henderson B, et al. The RpfC (Rv1884) atomic structure shows high structural conservation within the resuscitation-promoting factor catalytic domain. Acta Crystallogr Sect F. 2014;70:1022-6. https://doi.org/10.1107/S2053230X1401317X.

16. Maione V, Ruggiero A, Russo L, De Simone A, Pedone PV, Malgieri G, et al. NMR structure and dynamics of the resuscitation promoting factor RpfC catalytic domain. PLoS One. 2015;10:e0142807. http://dx.doi.org/10. 1371\%2Fjournal.pone. 0142807

17. Gupta RK, Srivastava BS, Srivastava R. Comparative expression analysis of rpflike genes of Mycobacterium tuberculosis H37Rv under different physiological stress and growth conditions. Microbiology. 2010;156:2714-22. https://doi.org/10.1099/mic.0.037622-0.

18. Raman S, Hazra R, Dascher CC, Husson RN. Transcription regulation by the Mycobacterium tuberculosis alternative sigma factor SigD and its role in virulence. J Bacteriol. 2004;186:6605-16. http://jb.asm.org/content/186/19/ 6605.abstract

19. Makinoshima H, Glickman MS. Regulation of Mycobacterium tuberculosis cell envelope composition and virulence by intramembrane proteolysis. Nature. 2005;436:406-9. https://doi.org/10.1038/nature03713

20. Rickman L, Scott $C$, Hunt DM, Hutchinson T, Menéndez MC, Whalan $R$, et al. A member of the CAMP receptor protein family of transcription regulators in Mycobacterium tuberculosis is required for virulence in mice and controls transcription of the rpfA gene coding for a resuscitation promoting factor. Mol Microbiol. 2005;56:1274-86. https:// doi.org/10.1111/j.1365-2958.2005.04609.x.

21. Shleeva M, Goncharenko A, Kudykina Y, Young D, Young M, Kaprelyants A. Cyclic amp-dependent resuscitation of dormant mycobacteria by exogenous free fatty acids. PLoS One. 2013;8:e82914. https://doi.org/10. 1371/journal.pone.0082914

22. Sharma AK, Chatterjee A, Gupta S, Banerjee R, Mandal S, Mukhopadhyay J, et al. MtrA, an essential response regulator of the MtrAB two-component system, regulates the transcription of resuscitation-promoting factor $B$ of Mycobacterium tuberculosis. Microbiology. 2015;161:1271-81. https://doi.org/ 10.1099/mic.0.000087.

23. Gupta RK, Srivastava R. Resuscitation promoting factors: a family of microbial proteins in survival and resuscitation of dormant mycobacteria. Indian J Microbiol. 2012;52:114-21. https://doi.org/10.1007/s12088-011-0202-6.
24. Hett EC, Chao MC, Steyn AJ, Fortune SM, Deng LL, Rubin EJ. A partner for the resuscitation-promoting factors of Mycobacterium tuberculosis. Mol Microbiol. 2007;66:658-68. https://doi.org/10.1111/j.1365-2958.2007.05945.x.

25. Hett EC, Chao MC, Deng LL, Rubin EJ. A mycobacterial enzyme essential for cell division synergizes with resuscitation-promoting factor. PLoS Pathog. 2008:4:e1000001. https://doi.org/10.1371/journal.ppat.1000001.

26. Hett EC, Chao MC, Rubin EJ. Interaction and modulation of two antagonistic cell wall enzymes of mycobacteria. PLoS Pathog. 2010;6:e1001020. https:// doi.org/10.1371/journal.ppat.1001020.

27. Choi H-G, Kim WS, Back YW, Kim H, Kwon KW, Kim J-S, et al. Mycobacterium tuberculosis RpfE promotes simultaneous Th1- and Th17-type T-cell immunity via TLR4-dependent maturation of dendritic cells. Eur J Immunol. 2015:45:1957-71. https://doi.org/10.1002/eji.201445329.

28. Målen H, Berven FS, Fladmark KE, Wiker HG. Comprehensive analysis of exported proteins from Mycobacterium tuberculosis H37Rv. Proteomics. 2007; 7:1702-18. https://doi.org/10.1002/pmic.200600853.

29. de Souza GA, Leversen NA, Målen H, Wiker HG. Bacterial proteins with cleaved or uncleaved signal peptides of the general secretory pathway. J Proteome. 2011;75:502-10.

30. Målen H, Pathak S, Søfteland T, de Souza GA, Wiker HG. Definition of novel cell envelope associated proteins in triton X-114 extracts of Mycobacterium tuberculosis H37Rv. BMC Microbiol. 2010;10:1-11. https://doi.org/10.1186/ 1471-2180-10-132.

31. Loraine J, Pu F, Turapov O, Mukamolova GV. Development of an in vitro assay for detection of drug-induced resuscitation-promoting-factordependent mycobacteria. Antimicrob Agents Chemother. 2016;60:6227-33. https://doi.org/10.1128/AAC.00518-16.

32. DiGiuseppe Champion PA, Cox JS. Protein secretion systems in mycobacteria. Cell Microbiol. 2007;9:1376-84. https://doi.org/10.1111/j.1462-5822.2007.00943.x.

33. Dammeyer $\mathrm{T}$, Tinnefeld $\mathrm{P}$. Engineered fluorescent proteins illuminate the bacterial periplasm. Comput Struct Biotechnol J. 2012;3:e201210013. https:// doi.org/10.5936/csbj.201210013.

34. Turapov O, O'Connor BD, Sarybaeva AA, Williams C, Patel H, Kadyrov AS, et al. Phenotypically adapted Mycobacterium tuberculosis populations from sputum are tolerant to first line drugs. Antimicrob Agents Chemother. 2016. 60:2476-83. https://doi.org/10.1128/AAC.01380-15.

35. Winterflood CM, Ewers H. Single-molecule localization microscopy using mCherry. ChemPhysChem. 2014;15:3447-51. https://doi.org/10.1002/cphc.201402423.

36. Subach FV, Patterson GH, Manley S, Gillette JM, Lippincott-Schwartz J, Verkhusha W. Photoactivatable mCherry for high-resolution two-color fluorescence microscopy. Nat Methods. 2009;6:153-9. https://doi.org/10. 1038/nmeth.1298.

37. Hu L, Vecchiarelli AG, Mizuuchi K, Neuman KC, Liu J. Directed and persistent movement arises from mechanochemistry of the ParA/ParB system. Proc Natl Acad Sci. 2015;112:E7055-64. https://doi.org/10.1073/pnas.1505147112.

38. Seeliger JC, Topp S, Sogi KM, Previti ML, Gallivan JP, Bertozzi CR. A riboswitch-based inducible gene expression system for mycobacteria. PLoS One. 2012;7:e29266. https://doi.org/10.1371/journal.pone.0029266.

39. Williams KJ, Joyce G, Robertson BD. Improved mycobacterial tetracycline inducible vectors. Plasmid. 2010;64:69-73. https://doi.org/10.1016/.plasmid. 2010.04.003.

40. Joyce G, Williams KJ, Robb M, Noens E, Tizzano B, Shahrezaei V, et al. Cell division site placement and asymmetric growth in mycobacteria. PLoS One. 2012;7:e44582. https://doi.org/10.1371/journal.pone.0044582.

41. Parish T, Stoker NG. Electroporation of mycobacteria. In: Parish T, Stoker NG, editors. Mycobacteria protocols. Totowa: Humana Press; 1998. p. 129-44. https://doi.org/10.1385/0-89603-471-2:129.

42. Schindelin J, Arganda-Carreras I, Frise E, Kaynig V, Longair M, Pietzsch T, et al. Fiji: an open-source platform for biological-image analysis. Nat Meth 2012;9:676-82. https://doi.org/10.1038/nmeth.2019.

43. Gasteiger E, Hoogland C, Gattiker A, Duvaud S, Wilkins MR, Appel RDBA. Protein identification and analysis tools on the ExPASy server. In: Walker JM, editor. The proteomics protocols handbook. Humana press; 2005. p. 571-607.

44. Miles AA, Misra SS, Irwin JO. The estimation of the bactericidal power of the blood. J Hyg (Lond). 1938;38:732-49. http://www.ncbi.nlm.nih.gov/pmc/ articles/PMC2199673/

45. Blodgett R. U.S. Food and Drug Administration. Bacteriological analytical manual, appendix 2: most probable number from serial dilutions. https://www. fda.gov/food/foodscienceresearch/laboratorymethods/ucm 109656.htm. 